\section{PNEUMOCOCCAL MENINGITIS.}

\author{
BY
}

C. WORSTER-DROUGHT, M.B.CANTAB., Temporary Captain R.A.M.C. OFFICER-IN-CHARGE CEREBRO-SPINAL FEVER WARD, ETC., ROTAL
HERBERT HOSPITAL, $\triangle N D$

ALEX. MILLS KENNEDY, M.D.GLAs., TEMPORARY Captain R.A.M.C..

BACTERIOLOGIST, ROYAL HERBEIT HOSPITAL

The following observations are based upon a study of nine cases of pneumococcal meningitis which have come under our direct care, the ages of the patients varying between the wide limits of three months and forty-one years. In five cases the disease was the so-called primary form-that is, no lesion conceivably due to active pneumococcal in. fection was discovered elsewhere. Thiis diagnosis; in three cases; was confirmed by post-mortem examination; in the remaining two an autopsy was not obtainable, but, clinically, no other lesion could be detected. In four cases meningitis occurred as a secondary infection.

\section{Primary Meningrtis.}

Of the five cases, two were infants, aged three and four months respectively, one was a boy of 11 , and two were adults aged 22 and 41 . The adults had had catarrhal "colds" for three or four days, followed by severe head. ache, prior to the appearance of meningeal symptoms, while the younger infant had had sudden convulsions. Following the appearance of headache, vomiting occurred in four of the five cases, and delirium rapidly supervened in the two aduits.

\section{SECondary Meningitis}

Of the four cases in which meningitis occurred as a secondary infection to a focus elsewhere, in two the primary lesion was a lobar pneumonia, in one middle-ear disease, and, in the last, an empyema of the sphenoidal sinus. The number of cases of pneumonia in which a secondary meningitis occurs is probably less than 2 per cent.

Lobar Pneumonia. - The first case, that of a child aged 14 months, was admitted to hospital with a temperature of $103^{\circ} \mathrm{F}$. pulse 140 , and respirations 80 . The only physical sign was dis tinctly impaired resonance over the upper lobe of the left lung, with -somerwhat diminished breath sounds; on the following day, however, bronchial breathing was present over this area, day, however, of the left lung. Neck rigidity was also present, the head show. of the left lung. Neck rigidity was also present, the lead show. ing a tendency to retraction, and the fontanelle increased internal tension. Lumbar puncture yielded a turbid fluid, diplococci; on culture a growth of pneumococci was obtained.

The second case, a girl aged 5 years, was first seen with an ordinary lobar pueumonia affecting the lower lobe of the right lung. On the fourth day of illness extreme restlessness and irritability were noticed; neck rigidity and Kernig's sign were elicited, and lumbar puncture showed a turbid fluid containivg elicited, and lum
pneumococci.

IIddle-ear Disease.-The patient, a man aged 23, had had an ear discharge eight months previously, which persisted for a few weeks and then ceased. Five days before admission to hospital he complained of severe headache, and the discharge $r \in c u r r e d ~ n e x t$ day. Two days later he vomited and became delirious the same evening.

Empyema of Sphenoidal Sinus.-In this patient, a man aged 26, headache had been present for a fow days when one morning 26, headache had been present for a few days when one morning it became increasingly severe and he vomited. During the He had maxle no complaint of any symptoms referable to a pathological nasal or post-nasal condition, and the empyema of the sphenoidal sinus was discovered only upon post-mortem examination.

Course.

From the day of onset the course of the disease varied from three to fifteen days' duration in different cases. With two adults, aged 22 and 41 , both cases of the primary form confirmed by post-mortem examination, the course of meningitis was extremely acute, lasting only three days; the temperatures were never below $101^{\circ}$, and often reached $104^{\circ}$. Five other cases were also acute; the one in which, post mortem, the sphenoidal sinus was found filled with pus died four days after the commencement of headache. Both the children in whom meningitis occurred secondarily to lobar pneumonia lived only two days after the appearance of meningeal symptoms. In none of the above cases did any lasting improvement follow the eracuation of cerebro. spinal fluid from the intrathecal space. A man aged 23, in whom meningitis occurred secondarily to otitis media, ran a course of nine days' duration, being continually delirious in spite of the daily withdrawal of 55 to $100 \mathrm{c.cm}$. of cerebro-spinal fluid, before sinking into coma. An infant of three months also continued for eight days acutely ill and with occasional convulsions. The courses of two other cases, both primary forms, were somewhat subacute; one was an infant, aged 4 months, who lived for twelve days with retracted head, arched spine, and occasional vomiting. Towards the end convulsions were frequent. The second subacute case is deserving of special mention.

The patient, a boy aged 11 years, complained of a headache and appeured generally unwell; three days later he vomited and seemed somewhat drowsy. For four days following this his condition remained about the same, the temperature remitting between $101^{\circ}$ and $104^{\circ}$. When seen on the fifth day his mental condition was quite normal, neck rigidity moderate, and Kernig's sign positive. 'The heart, lungs, ears, and nose showed no abnormality. Lumbar puncture was then instituted the fluid obtained being slightly opalescent and under moderate pressure ; direct examination of films showed the presence of numerous polymorphonuclear cells but no organisms. On culture, however, a growth of pneumococci was obtained. Lumbar puncture was continued until the day before death which occurred on the fifth day of illness; the withdrawal of 30 to $50 \mathrm{c.cm}$. of cerebro-spinal fluid was invariably followed by $\mathrm{a}$ fall in temperature, usually to normal or $99^{\circ}$. Throughout, the patient complained of headache of varying intensity. His condition continued with little or no alteration, either in the condition continued with little or no alteration, either in the
clinical signs or in the microscopical characters of the cerebrospinal tluid, until the eleventh day. Muscular rigidity was then more marked, and during the evening he became delirious and ircontinent for the first time. Lumbar puncture next day revealed a purulent fluid, which on microscopical examination showed numerous Gram-positive lanceolate diplococci; this was the first occasion on which organisms had been seen, in spite of prolonged search, on direct examination. Delirium and restlessness increased, floccilation and carphology appeared, and two days later the patient died.

Srmptoms and Signs.

Temperature.-In the seven acute cases the temperature generally remained at a high level, varying between $101^{\circ}$ and $104^{\circ}$, and seldom indeed falling below $100^{\circ}$. Lumbar puncture was without effect on the temperature in five of these cases ; in one infant, a primary form, it was occasionally followed by a fall of about two degrees $\left(103^{\circ}\right.$ to $\left.101^{\circ}\right)$, and in a second patient, with meningitis secondary to otitis media, the initial lumbar puncture produced a fall from $104^{\circ}$ to $1000^{\circ}$ Subsequent punctures had little or no effect on the pyrexia, although large amounts (85 to 100 c.cm.) of purulent fluid were obtained daily.

Of the two subacute cases, one has already been dealt with; the other, an infant of four months, admitted with a temperature of $102^{\circ}$, showed a fall to normal following the first lumbar puncture. After two days it gradually rose, with a few intermissions, to reach $103^{\circ}$ on the ninth day, thercafter never falling below $100^{\circ}$ and frequently reaching $105^{\circ}$. In all cases some rise of temperature occurred as death approached.

Pulse.-The pulse-rate was more frequent than is usual in either meningococcal or tuberculous meningitis. In one case only was it below 100 per minute on admission to hos. pital or when meningitis was discovered; in this case the pulse was 88 , but after the third day it gradually increased to 140 towards the end of the course. After the first few days the subacute case, aged 11, running a course of fifteen days, showed a pulse-rate varying between 84 and 96, with an increase to 100 to 120 during the last three days. In all other cases it varied between the limits of 120 and 160. Excepting in very acute cases, the pulse. rate showed a greater tendency to vary directly with the temperature than is usually seen in meningococcal meningitis.

Respiration.-With the exception of the two cases with lobar pneumonia, the respiratory rate was at first com. paratively slow, being 24 to 30 per minute in adults and 30 to 40 in children; the respirations increased in frequency towards the end of the course. In adults, both CheyneStokes respiration and Biot's respiration were observed.

Vomiting, etc.-Apart from that occurring at the onset, romiting was present in one case only, an infant aged 4 months. Profuse diarrhoea occurred at the onset in 
another infant, and, early in a course of nine days, in one adult.

Mental Condition.-Delirium, varying from incolerence to noisy restlessness, was the general rule in adults; in the cases continuing for only three days it was rapidly succeeded by coma. Screaming occasionally occurred in one infant, while, in a subacute case, the mental condition remained normal until the eleventh day, when delirium appeared, the patient dying on the fifteenth day. All cases died comatose.

Sphincters.-Excluding infants from consideration, retention of urine was present in two cases on admission, followed later by incontinence. The subacute case showed no change in the sphincter condition intil the eleventh day, when, with the onset of delirium, incontinence occurred. The remaining patients were incontinent throughout.

Cervical Muscles.-Pronounced retraction was present in the three infants, in the girl aged 6 years, and in one adult, a primary form, aged 22 . In all other cases there was rigidity of the neck muscles throughout the course, including the subacute case; no retraction, however, appeared in these cases.

Kernig's Sign.--In infants of under 2 years of age Kernig's sign is of no definite value in the diagnosis of meningitis; some authors state that it is a normal phenomenon at such an age, but this is incorrect. As a matter of interest, in one infant aged 13 months the sign was negative throughout the course of six days; in a second, aged 4 months, it was absent until the fourth day of illness, when it developed on the left side only, thus persisting until death on the twelfth day. In the last infant, aged 3 months, Kernig's sign was present on botl sides throughout a course of eight days. In all other cases it was well marked.

Spine.-Spinal rigidity was observed in all cases. The infant aged 4 montlis died on the twelfth day in extreme opisthotonos, which had gradually developed during the previous days. Both the other infants also showed some degree of opisthotonos.

Deep Reflexes.-In one patient only, aged 23, were the knee and ankle jerks absent throughout the course of meningitis; in all the infants they were at first slight, but disappeared during the last two or three days. In one adult they were distinctly exaggerated; with the supervention of coma they disappeared. Definite ankle clonus was not observed in any case.

Supcrficial Reflexes. - Plantars: Extensor responses were elicited in two patients only, aged 22 and 23 . Active withdrawal of the leg on plantar stimulation, so frequently observed in early cases of cerebro-spinal fever, was present in four cases. Ábdominals and epigastrics: 'These reflexes were absent throughout in all cases excepting three. They were slight at first in two adults, but disappeared later. In a subacute case they remained present until the elerenth day, disappearing with the onset of delirium and incontinence.

The reflexes, both deep and superficial, as a general rule, are directly proportional to the consciousness of the patient, being present in mild delirium and absent in more profound delirium and coma. The superficial reflexes disappear at an earlier stage than the knee and ankle jerks and, consequently, their absence, in the presence of other signs, rather favours diagnosis of meningitis.

Cranial Nerves.-The pupils were usually slightly dilated and reacted sluggishly to light; they were widely dilated throughout the illness in one adult dying on the fourth day. Inequality in size of the pupils was present in two cases only, an infant of 4 months and a boy aged 11 ; in the latter it developed on the twelfth day. 'This case was the only one in which definite strabismus was observed; it also appeared on the twelfth day, with an increase in meningeal symptoms, and was apparently due to an involvenient of the right sixth nerve. One case, a man aged 23 , had left facial paralysis, but this was due to involvement of the facial nerve by the infection from the middle ear, from which his meningitis originated; post mortem, pus was found in the Fallopian aqueduct.

Motor Disturbanices.-Convulsions occurred at the onset in two infants; both showed recurrences during courses of nine and twelve days respectively. Catching at imaginary objects and picking at the bedclothes (floccilation and cerphology) were present in tivo other cases.
Urinary Changes.-Glycosuria occurred on the eighth day of illness in a patient, aged 22, whose méningitis was secondary to chronic otitis media; he died on the ninth day. The glucose amounted to 5.8 grams per litrê and its presence was confirmed by the phenyl-hydrazine test. The occurrence of glycosuria during the course of meningitis has occasionally been noted; it is possible that it may be associated in some way with a disturbance of the floor of the fourth ventricle, such as pressure from within by distension of its cavity, or irritation of its ependymal lining. In the above case post-mortem examination showed the fourth ventricle to be somewhat distended with turbid fluid. Glycosuria also occurred in two cases of meningococcal meningitis coming under our care; in both, the appearance of glucose in the urine was coincident with symptoms of a degree of hydrocephalus. Both patients eventually recovered. Albuminuria was present in four adult cases.

Thic Cerebro-spinal Fluid.-A turbid fluid was obtained at the first lumbar puncture in the four cases of six years and under; the fluids were withdrawn on the first day of meningeal symptoms in the two cases secondary to pneumonia, and on the third and fourth day respectively in the two primary cases. Of the remaining five, the cerebrospinal fluid was definitely purulent in four adults, even at the first withdrawal, on the second or third day fol lowing the onset of illness. The first specimen of fluid from the last patient, aged 11 (the subacute case), was not obtained until the fifth day of illness; it was at first only slightly opalescent, and did not become purulent until the eleventh day. In the majority of cases there was a considerable increase in tension. In all, excepting the sub. acute case, pneumccocci were both seen on direct examination of stained films and obtained in culture from the first specimen of fluid withdrawn. In the exception no pneumococci were seen in films until the eleventh day. when for the first time the fluid was purulent; the organisms were obtained in culture, however, from that evacuated at the first withdrawal. Cytologically all fluids invariably showed numerous polymorphonuclear cells, and occasionally a few mononuclears. On testing with Fehling's solution, no glucose was ever detected in any of the cerebro-spinal fluids, with the single exception of that obtained from the subacute case, aged 11, on the first day of withdrawal (ifth day of illness). Following this, however, it disappeared next day, and remained absent.

IIokbid ANatory, etc.

Post-mortem examinations were made on seven of the nine cases; the two in which permission could not be obtained were apparently instances of the primary form of meningitis, in that no lesion was discoverable elsewhere, the chest, abdomen, ears, and accessory sinuses of the nose all appearing normal.

Primary Meningitis.

Of the three cases examined, two were infants aged three and four months respectively, and one was a man aged 41 years. In all cases the convolutions were flattened and well marked; purulent meningitis was present. In two cases greenish-yellow ; purulent meningitis was present. In two cases greenish-yellow
pus was more abundant over the convexity of the cerebrum, almost completely obscuring the cortex; there was less purulent exudate over the cerebellum, and very little in the pontine and medullary regions.

In the third case, an infant, there were patches of purulent exudate over the convexity of the cerebrum, with abundant greenish-yellow pus at the base, over and around the cere bellum, pons and medulla, being more plentiful anteriorly than posteriorly.

There was no pus found in the middle ears, in the frontal or sphenoidal sinuses, or in the ethmoidal air cells; nothing abnormal could be seen in the nasal fossae.

secondary Meningitis.

ia.-In both cases the meninges were very exudate over the cerebral cortex, with occasional pus along the course of the blood vessels. There was also considerable sero-purulent exudate over the pons and medulla, and, in one case, over the cerebellum also. In the infant aged thirteen months the upper lcbe of the left lung was consolidated; there was also oedema of the lower lobe, with adhesions at the base. The other case showed consolidation of the right lower lobe.

2. Chronic Otitis IIedia. - A well marked purulent meningitis was present, limited chiefly to the basal region of the brain and exterding over the pons, medulla, and cerebellum; pus could
be traced extending along the sides of the blood vessels up the be traced extending along the sides of the blood vessels up the
lateral aspects of the cerebrum. The right middle ear showed old-standing purulent otitis; pus also extended along the 
petrous bone to its internal tip, involving the inner ear, and appearing to be conveyed along the auditory nerve to the meninges. There was a small collection of thick pus lying meninges. There was and inner half of the petrous bone; in the Fallopian aqueduct pus was also found. No sinus thrombosis was present. Microscopical examination of films from both the was present. Microscopical examination of films from both the meningeal exudate and the pus in the midale ear showed pus cells with numerous pneumococci; examination of the disshown Stapfiylococcus aureus and a Gram-negative bacillus, but no pneumococci were seen or cultivated.

3. Empyema of Sphenoidal Sinus.-The autopsy showed well marked meningitis with massive fibrino-purulent exudation, limited almost wholly to the basal region of the brain; there were a few small patches of purulent exudate in the neighbourhood of the great longitudinal fissure. The sphenoidal sinus was found to be filled with pus and also the posterior ethmoida cells; the nasal fossae and other bony sinuses appeared normal. Films of the pus, both from the sphenoidal sinus and meninges, showed pneumococci.

In all cases the purulent exudate extended down the spinal cord. With regard to organs other than those already mentioned, the heart, liver, and kidneys invariably showed cloudy swelling; the spleen was occasionally enlarged and the pulp somewhat soft.

Diagnosis.

Meningitis having been diagnosed by the rigidity of the neck muscles, Kernig's sign, etc., its causative organism can only be determined by bacteriological examination of the arebro-spinal fluid. Even in the presence of pneu. monia meningitis is not necessarily pneumococcal, as, in occasional cases of cerebro-spinal fever, a bronchopneumonia may at first mask the meningeal symptoms. The majority of the cases described above were sent into hospital with a diagnosis of cerebro-spinal fever; it was only upon finding pneumococci in the cerebro-spinal fluid that their true nature was revealed. It is frequently possible to diagnose tuberculous meningitis clinically, but, at the present time; it is safer to regard any other examples of meningitis as meningococcal until proved otherwise.

\section{Prognosis}

The prognosis of pneumococcal meningitis is exceedingly grave, and it is doubtful if any severe cases ever recover. Hemenway ${ }^{1}$ reported the case of a clild, aged 2 years, suffering from pneumonia, who on the twelfth day dereloped head retraction and signs of meningitis. Lumbar puncture revealed a clear fluid under pressure, from which pneumococci were cultivated. The meningeal infection, however, must have been very mild, as the cerebro-spinal fluid was clear, showing no cellular reaction, and the case recovered. Four cases of recovery are mentioned by Broadbent, ${ }^{2}$ three being children and one a soldier. The latter does not appear to have lost consciousness, although the cerebro-spinal fluid was cloudy, and yielded a growth of pueumococci; the course of meningitis was of about twenty days' duration. Netter Shand ${ }^{3}$ and Carnarvon Brown ${ }^{4}$ have also recorded instances of recovery; that of the latter observer was a severe case, but unfortunately, beyond the statement that pneumococci were found in the cerebro-spinal fluid, nothing is said of the bacteriological findings. Shand's case was apparently very mild, the patient, aged 28 years, showing no deviation from normal consciousness, and recovering after a few days.

Treatuent.

The treatment of pneumococcal meningitis is at present most unsatisfactory. The nine cases recorded here serve to demonstrate the failure of repeated lumbar puncture. Each patient was punctured at least once daily, and in some cases every twelve hours, the subarachnoid space being drained on each occasion, until practically no more fluid would flow. As a result of the drainage the sym. ptoms were occasionally alleviated for a time, but the ultimate result was not affected. Vaccines may be of value, but it is seldom that a case lasts sufficiently long for vaccine therapy to have an effect.

We have found hexamine of no value in the treatment of meningitis. Since the chief therapeutic properties of the drug appear to be due to its oxidation into formaldehyde, which takes place only in an acid medium, it probably has little or no action in meningitis. In the course of the chemical examination of numerous samples of cerebro-spinal fluid from cases of 'meningitis, we have found less than 2 per cent. giving a very faintly acid reaction. The fluid is usually faintly alkaline, but occasionally neutral; the indicators used were litmus, phenolphthalein and methyl orange. Hexamine can be detected in the cerebro-spinal fluid within an hour of administration by the mouth, but the Burnam-Ramini test fails to demonstrate the presence of free formaldehyde.

Lamar; ${ }^{5}$ working with monkeys, showed that sodium oleate attacked and dissolved pneumococci, and, when consbined with a specific antipneumococcic serum, had a decided therapentic effect on pneumococcal meningitis, even when the organisms were multiplying in the blood stream. The action, however, is restricted to the par. ticular type of pneumococcus from which has been pro. duced the immune serum in the mixture of serum and sodium oleate. When the infecting pneumococcus was different in type from that represented by the immune serum, no therapeutic action resulted. When, therefore, a satisfactory polyvalent immune serum is forthcoming, the most promising method of treatment would seem to consist in the intrathecal injection of the antipneumococcic serum mixed with a solution of sodium oleate.

1 Hemenway Archives of PefERENCES

of Pediatrics, 1906, vol. xxiii, p. 276. ${ }^{2}$ Broadbent: BRITISH MEDiCAL JourNAL, October 28th, 1916, p. 586 . 3 Shand : tember 16th, 1916," '5 Lamar: Journ. Exper. Med., 1911, xiii, 1.

\section{AN INQUEST ON A LEG. \\ BY}

NORMAN PORRITT, M.R.C.S., L.R.C.P.LoND., CONBULTING SURGEON, HUDDURSFIELD ROYAL INFIRMARY.

ON April 6th, 1917, J. J., a master miller, disappeared from home. No trace of him was discovered until September 1st, when a fisherman found the romains of a leg in the river. In the hope that the rest of the body might be found the coroner ordered the river to be searched. This proving fruitless, the coroner decided to hold an inquest on the leg, and asked me to examine it and give evidence at the inquiry. 'The following notes of my examination may prove of interest.

On September 7th, five months and one day after the man disappeared, and six clays after the leg was found, I made the examination. On the foot was a boot, which was idlentified by the village shoemaker as one he had made for the missing man. Under the boot was a stocking foot of blue homespun. joined to the leg portion behind the heel, the greater joined to the leg portion behind the heel, the greater The missing man's wife identified the stocking, and also a bandage beneath it found round the ankle. It was clear, therefore, that the leg was that of the missing man.

The remains consisted of all the bones of the lower limb of the right side, more or less denuded of flesh. The femur was bare, except around the knee, from which the remains of the soft tissues, gradually tapering off, reached a point 4 in. above the middle of the patella. The head of the thigh bone, though denuded of cartilage, was undamaged; the neck was thick, denuded of cartilage, was undamaged; the neck was thick,
strong, and sprang obliquely from the shaft; and all the bony strong, and sprang obliquely from the shaft ; and all the bony
prominences and depressions were strongly marked. The bone was a compact whole, without trace of epiphyses. There was no antero-posterior bowing of the shaft. It was the bone of a man in the prime of life.

The patella was in position, but bare, and, though the fibula was covered and the structures of the calf were present, the anterior surfaces of the tibia were bare, except close to the knee and the ankle. The foot was attached to the limb only by the tendo Achillis. There was no trace of the ankle ligaments, and the articular surfaces of the joint denuded of cartilage were exposed. The foot was shrunken and black, but, protected by the boot, the skin had not disappeared. Over the heel the papillary ridges were visible to the naked eye. Across the sole along the roots of the toes.was a ragged cavity filled with soft soapy material, into which the structures had been converted.

The soft tissues of the calf and around the knee were dense and shrunken. An attempt was made to find the popliteal artery, to form an estimate of the man's age from the condition of its walls. But although the thick trunk of a nerve was isolated, the artery could not be found among the dense mass of homogeneous structure into which the soft tissues had been changed. Though the surface of the soft tissues was uniformly black, their snbstance was changed into dense white structures, and patches of white material studded their exterior.

There was no sign of old or recent injury, but on the upper third of the outer side of the femur the round shaft had been worn flat. The flat, slightly excavated surface was exquisitely smooth, and could have been caused only by long-continued friction, such as would occur if the limb had been caught and nipped, and swayed sbout by the current of the river. With the foot in its natural position the limb measured from the top 